\title{
"Piece" of mind: End of life in the intensive care unit Statement of the Belgian Society of Intensive Care Medicine
}

End-of-life decisions are common in the intensive care unit (ICU) with most deaths occurring in the ICU now preceded by a decision to limit treatment [1-4]. However, it is important to define the degree of "limitation of therapy." Restricting discussion to nonintervention in case of cardiac arrest-the "not to be resuscitated" or "do not resuscitate" order-is not sufficient. Other treatments must also be included in the global term limitation of therapy, including, for example, not applying mechanical ventilation in case of respiratory failure, not giving renal replacement therapy in case of acute renal failure, or not administering adrenergic support in case of acute circulatory shock. In this context, the principle of proportionality of care, as clearly defined in a French law [5], is an important concept, stating that therapies should only be administered in proportion to expected benefits. Hence, therapies that will act solely to artificially prolong life should not be started or should be discontinued.

Belgium [6,7] has specific laws dealing with euthanasia in terminally ill patients, but less than $1 \%$ of all deaths (approximately 1000 deaths/105000 total deaths per year in Belgium) occur as a result of requested euthanasia. Discontinuing therapies at the end of life in the ICU is performed in more than 10 times $(>10000$ deaths a year) the number of individuals who die under the conditions of the euthanasia law. Indeed, critically ill patients dying in the ICU are usually not in a position to request euthanasia. As a result, in Belgium, there is uncertainty about the legal consequences of starting a process that will result in death in the ICU. Although forgoing futile treatment is not against current Belgian legislation, difficulty can arise when the purpose of the drugs used for comfort and pain relief in end-of-life management is misconstrued as deliberate use to speed the dying process [8]. This statement paper, developed by members of the Belgian Society of Intensive Care Medicine Council, is not about giving analgesics or sedative agents to combat pain or agitation, nor about the so-called double effect, wherein analgesics given to alleviate pain may have the adverse effect of shortening the dying process. The discussion here is about the administration of sedative agents with the direct intention of shortening the process of terminal palliative care in patients with no prospect of a meaningful recovery. Importantly, many of the issues discussed may seem obvious and may even reflect current practice; nevertheless, in view of the emotive, ethical and potential legal nature of these issues, we believe that there is a need for them to be clearly stated. Moreover, we explain our belief in the concept that shortening the dying process by administering sedatives beyond what is needed for patient comfort can be not only acceptable but in many cases desirable.

\footnotetext{
The authors have no conflicts of interest to declare in relation to this article.
}

\section{Three generally well-accepted principles:}

1. Suffering should be avoided at all times. When the intensive care team reaches a consensus that current treatment no longer has any meaningful perspective and/or is disproportionate and/or is in conflict with advance directives, then it is ethically justified-and even appropriate-to stop this treatment. Indeed, such treatment would no longer bring benefit (principle of beneficence) and may even cause harm (principle of nonmaleficence) to the individual.

2. With the availability of modern organ support, most deaths in the ICU are preceded by a withhold/withdraw decision, often associated with increased doses of sedative/opioids agents with the intention to provide humane end-of-life care. It is ethically desirable to stop nonbeneficial treatment and to avoid continuing futile treatment. The Belgian Society of Intensive Care Medicine stated previously: "It is not only acceptable but necessary to stop active treatment in some patients who are arriving, irreversibly, at the end of their life" [9].

3. Relatives should be informed of prognosis and end-of-life decisions at all times.

Ten general complementary principles that we believe should be adopted:

1. As we have previously stated [9], although withdrawing a treatment sometimes seems more difficult than withholding, we believe there is no ethical or moral difference between the two; for example, the end result of discontinuing mechanical ventilation is the same as if it had not been started in the first place.

2. There is no clear ethical distinction between withholding/ withdrawing supportive therapy and increasing doses of sedative/opioid substances in patients in whom further treatment is no longer considered beneficial. Again, we previously stated: "Pragmatically, it is difficult to establish a coherent distinction between increasing analgo-sedation even substantially while maintaining life support, and discontinuing life support" [9].

3. Decisions to limit therapy must include all team members, not just the physicians. Every effort should also be made to involve all the physicians (including but not exclusively ICU physicians) who have cared for the patient. A consensus should be obtained for every end-of-life decision, although the decision remains the responsibility of the ICU physician. Once this consensus decision is made, all members of the team must apply the plan that has been decided on.

4. The plans for end-of-life care in each individual patient should be discussed with and understood by the relatives (or the 
patient's surrogate if one has been appointed). However, it must be made clear that the final decision is made by the care team and not by the relatives.

5. All end-of-life care planning should be clearly documented in the medical chart.

6. Shortening the dying process with use of medication, such as analgesics/sedatives, may sometimes be appropriate, even in the absence of discomfort, and can actually improve the quality of dying; this approach can also help relatives accompany their loved one through the dying process-such a decision should be made with due consideration for the wishes of family members.

7. Treatments may be stopped, but patient care is never discontinued.

8. Maintaining an individual's dignity must always remain a priority.

9. Through the entire process, the intention must not be interpreted as killing but as a humane act to accompany the patient at the end of his/her life.

10. The present document applies to children as well as to adults

Jean-Louis Vincent, MD, PhD Department of Intensive Care Erasme Hospital Université libre de Bruxelles Brussels, Belgium

E-mail address: jlvincen@ulb.ac.be

Miet Schetz, MD, PhD Department of Intensive Care University Hospital Leuven, Belgium

Jan J. De Waele, MD, PhD Department of Critical Care Medicine Ghent University and Ghent University Hospital Ghent, Belgium

Stéphan Clément de Cléty, MD Department of Intensive Care St Luc University Hospital Brussels, Belgium

Isabelle Michaux, MD, PhD Department of Intensive Care University Hospital Mont-Godinne, Belgium
Thierry Sottiaux, MD

Department of Intensive Care Clinique Notre Dame de Grace Gosselies, Belgium

Eric Hoste, MD, PhD Department of Critical Care Medicine Ghent University and Ghent University Hospital Ghent, Belgium

Didier Ledoux, MD PhD Department of Intensive Care University Hospital of Liège Belgium

Annick De Weerdt, MD Department of Intensive Care University Hospital of Antwerp Belgium

Alexander Wilmer, MD, PhD Department of Intensive Care University Hospital Leuven, Belgium

On behalf of the Belgian Society of Intensive Care Medicine http://dx.doi.org/10.1016/j.jcrc.2013.08.025

\section{References}

[1] Sprung CL, Cohen SL, Sjokvist P, et al. End-of-life practices in European intensive care units: the Ethicus Study. JAMA 2003;290:790-7.

[2] Brieva JL, Cooray P, Rowley M. Withholding and withdrawal of life-sustaining therapies in intensive care: an Australian experience. Crit Care Resusc 2009;11:266-8.

[3] Graw JA, Spies CD, Wernecke KD, et al. Managing end-of-life decision making in intensive care medicine-a perspective from Charite Hospital, Germany. PLoS One 2012;7:e46446

[4] Azoulay E Metnitz B, Sprung CL, et al. End-of-life practices in 282 intensive care units: data from the SAPS 3 database. Intensive Care Med 2009;35:623-30.

[5] Baumann A, Audibert G, Claudot F, et al. Ethics review: end of life legislation-the French model. Crit Care 2009;13:204.

[6] Vincent JL. End-of-life practice in Belgium and the new euthanasia law. Intensive Care Med 2006;32:1908-11.

[7] Deliens L, van der Wal G. The euthanasia law in Belgium and the Netherlands. Lancet 2003;362:1239-40.

[8] Sprung CL, Ledoux D, Bulow HH, et al. Relieving suffering or intentionally hastening death: where do you draw the line? Crit Care Med 2008;36:8-13.

[9] Ferdinande P, Berré J, Colardyn F, et al. La fin de la vie en médecine intensive. Réanimation 2001;10:340-1. 